Journal of Applied AnALysis

Vol. 11, No. 2 (2005), pp. 207-223

\title{
DOMINATED CONVERGENCE AND STONE-WEIERSTRASS THEOREM
}

\author{
J.-P. JURZAK \\ Received April 1, 2003 and, in revised form, September 10, 2004
}

\begin{abstract}
Let $\mathcal{C}(X ; \mathbb{R})$ the algebra of continuous real valued functions defined on a locally compact space $X$. We consider linear subspaces $\mathbb{A} \subset \mathcal{C}(X ; \mathbb{R})$ having the following property: there is a sequence $\left(\Phi_{j}\right)_{j \in \mathbb{N}}$ of positive functions in $\mathbb{A}$ with $\lim _{x \rightarrow \infty} \Phi_{j}(x)=+\infty$ for every $j \in \mathbb{N}$, such that $\mathbb{A}$ consists of functions $f \in \mathcal{C}(X ; \mathbb{R})$ bounded above for the absolute value by an homothetic of some $\Phi_{n}$ ( $n$ depends on each $f$ ). Dominated convergence of a sequence $\left(g_{n}\right)_{n \geq 1}$ in $\mathbb{A}$ is an estimation of the form $\left|g_{n}(x)-g(x)\right| \leq \varepsilon_{n}|h(x)|$ for all $x \in X$ and all $n \in \mathbb{N}$ where $g_{n}, g, h \in \mathbb{A}$ and $\varepsilon_{n} \rightarrow 0$ as $n \rightarrow \infty$. We extend the Stone-Weierstrass theorem to subalgebras or lattices $\mathbb{B} \subset \mathbb{A}$ and we show that the dominated convergence for sequences is exactly the convergence of sequences when $\mathbb{A}$ is endowed with a locally convex $(\mathcal{D} \mathcal{F})$-space topology.
\end{abstract}

\section{Preliminaries}

On a compact space $S$, the classical Stone-Weierstrass theorem states that suitable subalgebras or linear subspaces of $\mathcal{C}(S)$ (the space of continuous

2000 Mathematics Subject Classification. Primary 42A65, 46E05; Secondary 46A04, $46 \mathrm{~A} 17$.

Key words and phrases. Stone-Weierstrass, approximation, $\mathcal{D} \mathcal{F}$-spaces, Mackey convergence.

ISSN 1425-6908（C) Heldermann Verlag. 
real or complex valued functions on $S$ ) are dense in $\mathcal{C}(S)$ for the topology associated to the supremum norm

$$
\|f\|_{\infty}=\sup \{|f(t)| ; t \in S\} \quad \text { for } \quad f \in \mathcal{C}(S) .
$$

Various extensions of the Stone-Weierstrass theorem have been obtained with simplified proofs [2], [9], [12], and $C^{\infty}$ versions as in [1, Appendix B].

The purpose of this paper is to leave the context of bounded functions in order to deal with continuous bounded or unbounded real valued functions defined on a locally compact space $X$; this jump becomes possible by the use of the dominated convergence.

A sequence $\left(g_{n}\right)_{n \geq 1}$ of continuous functions defined on $X$ converges to a function $g$ defined on $X$ in a dominated way iff there exists a real sequence $\varepsilon_{n} \rightarrow 0$ and a continuous function $h$ defined on $X$ (in general $h$ is an unbounded function) such that

$$
\left|g_{n}(x)-g(x)\right| \leq \varepsilon_{n}|h(x)|
$$

for all $x \in X$ and all $n \in \mathbb{N}$.

The natural and simplest context of the dominated convergence assumes a given couple $(X ; \Phi)$ where $X$ is a locally compact space and $\Phi$ a fixed continuous real-valued (in general unbounded) strictly positive function defined on $X$. Let $\mathcal{C}(X ; \mathbb{R})$ be the space of continuous real valued functions defined on $X$; the set of $f \in \mathcal{C}(X ; \mathbb{R})$ with a growth on $X$ smaller in absolute value than some power (depending on $f$ ) of $\Phi$ is a real subalgebra $\mathcal{C}^{\Phi}(X)$ of $\mathcal{C}(X ; \mathbb{R})$. The dominated convergence of a sequence $\left(f_{n}\right) \in \mathcal{C}^{\Phi}(X)$ with limit $f \in \mathcal{C}^{\Phi}(X)$ becomes the existence of a suitable $\alpha>0$ and a real sequence $\varepsilon_{n} \rightarrow 0$ (depending on the $f_{n}$ 's and $f$ ) such that one gets an inequality of the form

$$
\left|f_{n}(x)-f(x)\right| \leq \varepsilon_{n} \Phi^{\alpha}(x)
$$

for all $x \in X$ and all $n \in \mathbb{N}$.

The article is organized as follows: in part one, we consider examples of classical power series for which the concept of dominated convergence appears to be a natural relation between the Taylor series and the function itself. Estimations so obtained apply directly to spectral theory in Hilbert spaces.

Part two is concerned with main properties of dominated convergence.

In part three, we see that dominated convergence allows a theorem of Stone-Weierstrass well-adapted for general functional analysis. In the case of a compact topological space $S$, all continuous functions defined on $S$ are dominated (up to an homothetic) by the constant function 1, thus convergence agrees with dominated convergence and corresponds to $\Phi=1$. In our context (Theorem 4.1), the theorem of Stone-Weierstrass works with 
the couple $(X, \Phi)$ where $\Phi$ is a fixed continuous unbounded real function defined on a locally compact space $X$.

In part four, we describe the locally convex topology associated to dominated convergence (Theorem 5.4): this topology is an inductive limit topology (not strict) of a sequence of Banach spaces. Contrary to current ideas, these spaces are far from being pathological [7] and are very adapted to the functional analysis (this is due to the order structure associated to the cone of positive functions).

Throughout this paper, $X$ is a locally compact space, $\mathcal{C}(X ; \mathbb{R})$ is the space of continuous real valued functions defined on $X$ and $\mathcal{C}^{b}(X ; \mathbb{R})$ denotes the subalgebra of $\mathcal{C}(X ; \mathbb{R})$ consisting of bounded functions on $X$.

1. A subset $\mathcal{S} \subset \mathcal{C}(X ; \mathbb{R})$ is separating (or is said to separate the points of $X$ ) if given $x, y \in X$ with $x \neq y$, there is $g \in S$ with $g(x) \neq g(y)$.

2. A subset $\mathcal{S} \subset \mathcal{C}(X ; \mathbb{R})$ is everywhere different from zero on $X$ (or is said to be non-vanishing on $X$ ) if given $x \in X$ there is $g \in \mathcal{S}$ with $g(x) \neq 0$.

\section{Simple facts}

The series $\sum_{n \geq 0} x^{n}$ is simply convergent for $x \in[0 ; 1[$ and

$$
\frac{1}{1-x}-\left(1+x+x^{2}+x^{3}+\cdots+x^{n-1}\right)=\frac{x^{n}}{1-x}=\frac{x^{n}(1-x)}{(1-x)^{2}} .
$$

Noting that $\sup _{x \in[0 ; 1[} x^{n}(1-x)=\varepsilon(n)$ tends to 0 as $n \rightarrow+\infty$, we get

$0 \leq \frac{1}{1-x}-\left(1+x+x^{2}+x^{3}+\cdots+x^{n-1}\right) \leq \frac{\varepsilon(n)}{(1-x)^{2}} \quad$ with $\lim _{n \rightarrow \infty} \varepsilon(n)=0$

(in fact $1 /[e(n+1)]<\varepsilon(n)<1 /(e n))$. Clearly, for $\alpha>0$ and $0 \leq x<1$

$$
0 \leq \frac{1}{1-x}-\left(1+x+x^{2}+x^{3}+\cdots+x^{n-1}\right) \leq \frac{\varepsilon_{1}(n)}{(1-x)^{1+\alpha}}
$$

where $\lim _{n \rightarrow \infty} \varepsilon_{1}(n)=0$. This type of convergence may be defined as a dominated convergence, the functions $1 /(1-x)^{2}$ and $1 /(1-x)^{1+\alpha}$ being the dominating functions $[0 ; 1[$.

We deduce, for $\alpha>0,0 \leq x<1$ :

$$
0 \leq-\ln (1-x)-\left(x+\frac{1}{2} x^{2}+\frac{1}{3} x^{3}+\cdots+\frac{1}{n} x^{n}\right) \leq \frac{\varepsilon_{2}(n)}{(1-x)^{\alpha}}
$$

where $\lim _{n \rightarrow \infty} \varepsilon_{2}(n)=0$. We note, for $0 \leq x<1,0 \leq y<1$, that

$$
\begin{aligned}
0 \leq & \frac{1}{(1-x)(1-y)}-\left(1+(x+y-x y)+(x+y-x y)^{2}+\cdots\right. \\
& \left.+(x+y-x y)^{n-1}\right)
\end{aligned}
$$




$$
\leq \frac{\varepsilon_{3}(n)}{((1-x)(1-y))^{1+\alpha}} \quad \text { with } \lim _{n \rightarrow \infty} \varepsilon_{3}(n)=0 .
$$

Choosing now the exponential function, one has, for $x>0$

$$
0 \leq e^{x}-\left(1+x+\frac{1}{2 !} x^{2}+\frac{1}{3 !} x^{3}+\cdots+\frac{1}{n !} x^{n}\right)=R_{n}(x)
$$

with

$$
R_{n}(x)=\frac{x^{n+1}}{n !} \int_{0}^{1}(1-t)^{n} e^{t x} d t=\frac{x}{n !} \int_{0}^{1}(1-t)^{n}\left(x^{n} e^{(t-1) x}\right) e^{x} d t .
$$

For $0<t<1$ and any $x \in[0 ;+\infty[$

$$
0 \leq x^{n} e^{(t-1) x} \leq \frac{n^{n}}{(1-t)^{n}} e^{-n}
$$

hence

$$
0 \leq R_{n}(x) \leq \frac{x e^{x}}{n !} \int_{0}^{1} n^{n} e^{-n} d t
$$

From

$$
\frac{n^{n} e^{-n}}{n !} \sim \frac{1}{\sqrt{2 \pi n}}
$$

we get

$$
0 \leq e^{x}-\left(1+x+\frac{1}{2 !} x^{2}+\frac{1}{3 !} x^{3}+\cdots+\frac{1}{n !} x^{n}\right) \leq \eta(n) x e^{x}
$$

where $\lim _{n \rightarrow \infty} \eta(n)=0$. We deduce, for $\alpha>0$ and $x>0$

$$
0 \leq e^{x}-\left(1+x+\frac{1}{2 !} x^{2}+\frac{1}{3 !} x^{3}+\cdots+\frac{1}{n !} x^{n}\right) \leq \eta_{1}(n) e^{(1+\alpha) x}
$$

with $\lim _{n \rightarrow \infty} \eta_{1}(n)=0$. Here, $x e^{x}$ and $e^{(1+\alpha) x}$ are dominating functions on $[0 ;+\infty[$.

This process of domination appears systematically in the commutative or non-commutative analysis. It allows general approximations of unbounded operators and more generally of continuous sesquilinear forms of spectral theory.

As an application, let $H$ be an Hilbert space, $\Delta$ be an unbounded self adjoint operator with dense domain $\operatorname{Dom}(\Delta)$ dense in $H$ and

$$
\Delta=\int \lambda d p_{\lambda} \quad \text { be its spectral decomposition. }
$$

Taking $f(x)=x$, we see, putting $\chi_{[-n, n]}=1$ for $-n \leq x \leq n$ and $\chi_{[-n, n]}=0$ elsewere, that $\left|f(x)-x \cdot \chi_{[-n, n]}\right| \leq(1 / n) x^{2}$ for all $x \in \mathbb{R}$. It follows, for all $v \in \operatorname{Dom}(\Delta)$

$$
\left\|\left(\Delta-\Delta_{n}\right) v\right\| \leq \frac{1}{n}\left\|\Delta^{2}(v)\right\| \quad \text { with } \quad \Delta_{n} \equiv \int_{-n}^{n} \lambda d p_{\lambda} .
$$


When $\Delta$ is a positive operator, we get:

$$
0 \leq\left|\left(\left(\Delta-\Delta_{n}\right) v, v\right)\right| \leq \frac{1}{n}\left|\left(\Delta^{2} v, v\right)\right| \quad \text { with } \quad \Delta_{n} \equiv \int_{0}^{n} \lambda d p_{\lambda} \geq 0
$$

for all $v \in \operatorname{Dom}(\Delta)$, where $(v, w)$ stands for the scalar product of vectors $v, w$ in $H$. We refer to [7] for a general non-commutative study.

\section{On dominated convergence}

Proposition 3.1. Let $X$ be a locally compact space, $f_{n}: X \rightarrow \mathbb{R}$ be an increasing sequence of continuous functions simply convergent to a continuous function $f: X \rightarrow \mathbb{R}$. Let $f^{*}: X \rightarrow \mathbb{R}$ be a continuous function such that

$$
f^{*}(x) \neq 0 \quad \forall x \in X \quad \text { and } \quad \lim _{x \rightarrow \infty} \frac{f(x)-f_{1}(x)}{f^{*}(x)}=0 .
$$

Then, there exists a real sequence $\varepsilon_{n} \rightarrow 0$ such that

$$
\left|f_{n}(x)-f(x)\right| \leq \varepsilon_{n}\left|f^{*}(x)\right|
$$

for all $x \in X$ and all $n \in \mathbb{N}$.

Assuming that condition $f^{*}(x) \neq 0$ for all $x \in X$ is satisfied, we note that the two conditions

$$
\lim _{x \rightarrow \infty} \frac{f_{1}(x)}{f^{*}(x)}=0 \quad \text { and } \quad \lim _{x \rightarrow \infty} \frac{f(x)}{f^{*}(x)}=0
$$

imply condition (3.1) as well as

$$
f_{n}(x) \geq 0 \quad \forall x \forall n \quad \text { and } \quad \lim _{x \rightarrow \infty} \frac{f(x)}{f^{*}(x)}=0 .
$$

Proof. Let $g_{n}(x)=f_{n}(x)-f_{1}(x)$ for $n \in \mathbb{N}$ and $g(x)=f(x)-f_{1}(x)$. Then $g_{n}: X \rightarrow \mathbb{R}$ is an increasing sequence of positive continuous functions simply convergent to the continuous function $g: X \rightarrow \mathbb{R}$. From $\lim _{x \rightarrow \infty} g(x) / f^{*}(x)=0$ and $0 \leq g_{n}(x) \leq g(x)$, we get for any $n$

$$
\lim _{x \rightarrow \infty} \frac{g_{n}(x)}{f^{*}(x)}=0
$$

Functions $g_{n} / f^{*}$ and $g / f^{*}$ extend continuously to the Alexandroff compactification $\bar{X}=X \cup \infty$ of $X$ by choosing the zero value at the infinity point. By Dini's theorem applied to the increasing sequence $\left(g_{n} / f^{*}\right)_{n \in \mathbb{N}}$ of continuous functions, we get the uniform convergence on the compact space $\bar{X}$ which is the estimation

$$
\left|\frac{f_{n}(x)-f_{1}(x)}{f^{*}(x)}-\frac{f(x)-f_{1}(x)}{f^{*}(x)}\right| \leq \varepsilon_{n}
$$


for a suitable sequence $\varepsilon_{n} \rightarrow 0$ : this proves (3.2).

Proposition 3.2. Let $X$ be a locally compact space and $\sum_{n \geq 0} u_{n}=u(x)$ be an absolutely simply convergent series of continuous functions $u_{n}: X \rightarrow \mathbb{R}$.

We assume that $\widetilde{u}(x)=\sum_{n \geq 0}\left|u_{n}(x)\right|$ is a continuous function on $X$.

1. Let $u^{*}: X \rightarrow \mathbb{R}$ be a continuous function satisfying

$$
u^{*}(x) \neq 0 \quad \forall x \in X \quad \text { and } \quad \lim _{x \rightarrow \infty} \frac{\widetilde{u}(x)}{u^{*}(x)}=0 .
$$

Then, there exists a real sequence $\varepsilon_{n} \rightarrow 0$ such that

$$
\left|\sum_{i=0}^{i=n} u_{i}(x)-u(x)\right| \leq \varepsilon_{n}\left|u^{*}(x)\right|
$$

for all $x \in X$ and all $n \in \mathbb{N}$.

2. Let $\sum_{n \geq 0} v_{n}=v(x)$ be a second simply convergent series of continuous functions $v_{n}: X \rightarrow \mathbb{R}$ with a real sequence $\varepsilon_{n}^{*} \rightarrow 0$ as $n \rightarrow \infty$, such that, for all $x \in X, n \in \mathbb{N}$

$$
\left|\sum_{i=0}^{i=n} v_{i}(x)-v(x)\right| \leq \varepsilon_{n}^{*}\left|v^{*}(x)\right|
$$

for some continuous function $v^{*}: X \rightarrow \mathbb{R}$. We put, for $k \in \mathbb{N}, x \in X$

$$
w_{k}(x)=\sum_{j=0}^{j=k} u_{j}(x) v_{k-j}(x) .
$$

Then, there exists a real sequence $\eta_{n} \rightarrow 0$ as $n \rightarrow \infty$ such that

$$
\left|\sum_{i=0}^{i=n} w_{i}(x)-u(x) v(x)\right| \leq \eta_{n}\left|u^{*}(x) v^{*}(x)\right|
$$

for all $x \in X, n \in \mathbb{N}$.

Proof. For part 1), one has

$$
\left|\sum_{i=0}^{i=n} u_{i}(x)-u(x)\right| \leq \sum_{i>n}\left|u_{i}(x)\right|=\widetilde{u}(x)-\sum_{i=0}^{i=n}\left|u_{i}(x)\right| .
$$

With $f_{n}(x)=\sum_{i=0}^{i=n}\left|u_{i}(x)\right|$ for $n \in \mathbb{N}$ and $f(x)=\widetilde{u}(x)$, we easily see that condition (3.4) is satisfied with $f^{*}(x)=u^{*}(x)$. Thus,

$$
0 \leq \widetilde{u}(x)-\sum_{i=0}^{i=n}\left|u_{i}(x)\right| \leq \varepsilon_{n}\left|u^{*}(x)\right|
$$

implies (3.6). 
For part 2), our hypothesis implies

$$
\left|\sum_{i=0}^{i=n} \frac{u_{i}(x)}{\left|u^{*}(x)\right|}-\frac{u(x)}{u^{*}(x) \mid}\right| \leq \varepsilon_{n} \quad \text { and } \quad\left|\sum_{i=0}^{i=n} \frac{v_{i}(x)}{\left|v^{*}(x)\right|}-\frac{v(x)}{\left|v^{*}(x)\right|}\right| \leq \varepsilon_{n}^{*} .
$$

It follows that the product $\sum_{k \geq 1} c_{k}(x)$ of the two uniformly convergent series

with

$$
\sum_{k \geq 0} u_{k}(x) /\left|u^{*}(x)\right| \text { and } \sum_{k \geq 0} v_{k}(x) /\left|v^{*}(x)\right|
$$

$$
c_{k}(x)=\sum_{j=0}^{j=k} \frac{u_{j}(x)}{\left|u^{*}(x)\right|} \frac{v_{k-j}(x)}{\left|v^{*}(x)\right|}=\frac{w_{k}(x)}{\left|u^{*}(x) v^{*}(x)\right|}
$$

(the first series is absolutely and uniformly convergent) is uniformly convergent to the product $u(x) v(x) /\left|u^{*}(x) v^{*}(x)\right|$ which is assertion (3.8).

Proposition 3.3. Let $X$ be a locally compact space and $\left(u_{n}\right)_{n \geq 0}$ be a decreasing sequence of positive continuous functions $u_{n}: X \rightarrow \mathbb{R}$ such that $\lim _{n \rightarrow+\infty} u_{n}(x)=0$ for $x \in X$.

For $x \in X$, let $\sum_{n=0}^{+\infty}(-1)^{n} u_{n}(x)=u(x)$ assumed continuous on $X$ and $u^{*}: X \rightarrow \mathbb{R}$ be a continuous function satisfying

$$
u^{*}(x)>0 \quad \forall x \in X \quad \text { and } \quad \lim _{x \rightarrow \infty} \frac{u(x)}{u^{*}(x)}=0 .
$$

Then, there exists a real sequence $\varepsilon_{n} \rightarrow 0$ such that

$$
\left|\sum_{i=0}^{i=n}(-1)^{i} u_{i}(x)-u(x)\right| \leq \varepsilon_{n} u^{*}(x)
$$

for all $x \in X$ and all $n \in \mathbb{N}$.

Proof. We put $w_{i}(x)=u_{2 i}(x)-u_{2 i+1}(x)$ for $i \geq 0, x \in X$ : as $w_{i}(x) \geq 0$, one has $u(x) \geq 0$ and we may assume $u(x)>0$ for all $x \in X$. The sequence

$$
S_{2 n}(x)=\sum_{i=0}^{i=2 n+1}(-1)^{i} \frac{u_{i}(x)}{u^{*}(x)}=\sum_{i=0}^{i=n} \frac{w_{i}(x)}{u^{*}(x)}
$$

is an increasing sequence of positive continuous functions simply convergent to $u(x) / u^{*}(x)$ for all $x \in X$. It follows that

$$
\lim _{x \rightarrow \infty} \frac{w_{i}(x)}{u^{*}(x)}=0 \quad \forall i \geq 0 \quad \forall x \in X .
$$

Let $\bar{X}=X \cup \infty$ be the Alexandroff compactification of $X$. Functions $S_{2 n}(x)$ and $u(x) / u^{*}(x)$ have zero limit as $x \rightarrow \infty$, hence extend continuously at 
$x=\infty$ by the settings $S_{2 n}(\infty)=u(x) / u^{*}(\infty)=0$. By Dini's theorem, there exists a real sequence $\varepsilon_{2 n+1} \rightarrow 0$ such that

$$
\left|S_{2 n}(x)-\frac{u(x)}{u^{*}(x)}\right| \leq \varepsilon_{2 n+1} .
$$

Working similarly with the sequence

$$
S_{2 n+1}(x)=\sum_{i=0}^{i=2 n}(-1)^{i} \frac{u_{i}(x)}{u^{*}(x)}
$$

we get a sequence $\varepsilon_{2 n} \rightarrow 0$ which leads to estimation (3.10).

Corollary 3.4. For $r \in \mathbb{R}$ and $0 \leq x<1$ and $\alpha>0$

$$
0 \leq \frac{1}{(1-x)^{r}}-\sum_{j=0}^{j=n-1} C_{-r}^{j}(-x)^{j} \leq \frac{\varepsilon(n)}{(1-x)^{r+\alpha}} .
$$

For $-1<x \leq 0$

$$
0 \leq \frac{1}{(1-x)^{r}}-\sum_{j=0}^{j=n-1} C_{-r}^{j}(-x)^{j} \leq \frac{\varepsilon_{1}(n)}{(1-x)^{\alpha}} .
$$

For $0 \leq x$ and $\alpha>0$

$$
0 \leq e^{-x}-\sum_{j=0}^{j=n-1}(-1)^{j} \frac{x^{j}}{j !} \leq \eta(n) e^{\alpha x}
$$

with $\lim _{n \rightarrow \infty} \varepsilon(n)=\lim _{n \rightarrow \infty} \varepsilon_{1}(n)=\lim _{n \rightarrow \infty} \eta(n)=0$.

For $-1<x \leq 0$ and $\alpha>0$, formula (3.13) with $r=1$ expands as

$$
\left|\frac{1}{1-x}-\left(1+x+x^{2}+\cdots+x^{n-1}\right)\right| \leq \frac{\varepsilon(n)}{(1-x)^{\alpha}} \quad \text { with } \lim _{n \rightarrow \infty} \varepsilon(n)=0 .
$$

Comparing with formula (2.1), we see that the singularity of $\sum_{n} x^{n}$ at $x=-1$ is weaker than at $x=1$.

Proof. For $r>0$, coefficients $C_{-r}^{j}$ are positive and formula (3.12) follows from formula (3.6). When $r \in \mathbb{Z}$ with $r<0$, formula (3.12) amounts to a finite summation, thus the case $r<0$ follows from multiplication of series (part 2) of Proposition 3.2.

For formula (3.13), we observe that, for $r>0$ the $y$-series $\sum_{j} C_{-r}^{j} y^{j}$ with $y=-x$ satisfies the hypothesis of Proposition 3.3 (for large $n$, the coefficients in the series are alternatively positive and negative) and for $r<0$, we apply part 2) of Proposition 3.2. 
Concerning the function $e^{-x}$, one has

$$
e^{-x}-\left(1-x+\frac{1}{2 !} x^{2}-\frac{1}{3 !} x^{3}+\cdots+(-1)^{n} \frac{1}{n !} x^{n}\right)=R_{n}(x)
$$

with

$$
\left|R_{n}(x)\right|=\frac{x^{n+1}}{n !} \int_{0}^{1}(1-t)^{n} e^{-t x} d t=\frac{\eta^{n+1}}{n !} y^{n+1} \int_{0}^{1}(1-t)^{n} e^{-t \eta y} d t
$$

where $y=x / \eta$. The evaluation of the extremum of $y^{n+1} e^{-t \eta y}$ on $[0 ;+\infty[$ and Stirling formula lead, for $\eta>0$ and $x>0$, to

$$
0 \leq e^{-x}-\left(1-x+\frac{1}{2 !} x^{2}-\frac{1}{3 !} x^{3}+\cdots+(-1)^{n} \frac{1}{n !} x^{n}\right) \leq \eta_{1}(n) e^{\eta x}
$$

with $\lim _{n \rightarrow \infty} \eta_{1}(n)=0$.

Lemma 3.5. Let $f(z)=\sum_{i \geq 0} b_{i} z^{i}$ be a power series with $b_{n} \geq 0$ for all $n \geq 1, f(0) \neq 0$ and $R>0$ be the radius of convergence. Let $\eta>0$ be fixed. Then there exists a sequence $\varepsilon_{n} \rightarrow 0$ as $n \rightarrow+\infty$ such that one has

$$
0 \leq f(x)-\sum_{i=0}^{i=n} b_{i} x^{i} \leq \varepsilon_{n} f^{1+\eta}(x)
$$

for all $x \geq 0$ and all $n \geq 0$. For $z \in \mathbb{C}$, we get

$$
\left|f(z)-\sum_{i=0}^{i=n} b_{i} z^{i}\right| \leq \varepsilon_{n} f^{1+\eta}(|z|) .
$$

The case $f(0)=0$ is easily handled with $f$ replaced by $f+c$ with $c>0$ since

$$
f(x)-\sum_{i=1}^{i=n} b_{i} x^{i}=(f(x)+c)-\left(c+\sum_{i=1}^{i=n} b_{i} x^{i}\right) .
$$

Proof. We assume that $f(z)$ is not a polynomial. Let $R$ be finite. If $\sum_{i=0}^{\infty} b_{i}<+\infty$, by Abel's lemma, the sequence of functions $f_{n}(x)=$ $\sum_{i=0}^{i=n} b_{i} x^{i}$ is an increasing sequence of continuous functions converging simply on $[0 ; R]$ to the continuous function $f(x)$, therefore the convergence is uniform on that interval by Dini's theorem. For a given $\varepsilon>0$, there is an $N$ such that, for $x \in[0 ; R]$ and $n \geq N$

$$
0 \leq f(x)-\sum_{i=0}^{i=n} b_{i} x^{i} \leq \varepsilon b_{0}^{1+\eta} \leq \varepsilon f^{1+\eta}(x)
$$


showing (3.15). Due to

$$
\left|f(z)-\sum_{i=0}^{i=n} b_{i} z^{i}\right| \leq \sum_{i=n+1}^{\infty} b_{i}|z|^{i}
$$

we get (3.15).

When $\sum_{i=0}^{\infty} b_{i}=+\infty$ and $R$ finite, we obtain

$$
0<f_{n}(x) \leq f(x) \quad \forall x \geq 0 \text { and } \lim _{x \rightarrow R} \frac{f(x)}{f^{1+\eta}(x)}=0
$$

since $\lim _{x \rightarrow R} f(x)=+\infty$ (this property was proved by Abel). Now, $f(x)>$ 0 for $x \in[0 ; R[$, thus Proposition 3.1 applied and yields

$$
0 \leq f(x)-\sum_{i=0}^{i=n} b_{i} x^{i} \leq \varepsilon_{n} f^{1+\eta}(x)
$$

for all $0 \leq x<R$, all $n \geq 1$ and a suitable real sequence $\varepsilon_{n} \rightarrow 0$ as $n \rightarrow+\infty$.

When $f$ is entire, it is clear that $\lim _{x \rightarrow+\infty} f(x)=+\infty$ and the same proof establishes (3.15). Other assertions follows from (3.17).

Corollary 3.6. Let $f(z)=\sum_{i \geq 0} a_{i} z^{i}$ be a power series with $a_{i} \in \mathbb{C}$ and $f_{n}(z)=\sum_{i=0}^{i=n} a_{i} z^{i}$. Let $R$ be the radius of convergence of $f$. Then, there exists a power series $f^{*}$ with radius of convergence $\geq R$ and a real sequence $\varepsilon_{n} \rightarrow 0$ as $n \rightarrow+\infty$ such that one has

$$
\left|f(x)-\sum_{i=0}^{i=n} a_{i} x^{i}\right| \leq \varepsilon_{n} f^{*}(x)
$$

for all $x \geq 0$ and all $n \geq 0$. For $z \in \mathbb{C}$, we get

$$
\left|f(z)-\sum_{i=0}^{i=n} a_{i} z^{i}\right| \leq \varepsilon_{n} f^{*}(|z|) .
$$

Indeed, $f$ is a linear combination of series satisfying the preceeding lemma and we choose $\eta=1$. We note that $f^{*}$ is an entire function in the complex plane when $R=+\infty$.

\section{The Stone-Weierstrass theorem}

As mentioned previously, $X$ is a locally compact space, $\mathcal{C}(X ; \mathbb{R})$ is the space of continuous real valued functions defined on $X$ and $\mathcal{C}^{b}(X ; \mathbb{R})$ denotes the subalgebra of $\mathcal{C}(X ; \mathbb{R})$ consisting of bounded functions on $X$.

For $\Phi: X \rightarrow \mathbb{R}$ continuous strictly positive function everywhere defined on $X, \mathcal{C}^{\Phi}(X)$ stands for the real subalgebra of $\mathcal{C}(X ; \mathbb{R})$ consisting of functions 
$f \in \mathcal{C}(X ; \mathbb{R})$ bounded above for the absolute value by a multiple of some power (depending on $f$ ) of $\Phi$. We put:

$$
\mathcal{C}_{0}(X ; \mathbb{R})=\left\{f \in \mathcal{C}(X ; \mathbb{R}) ; \lim _{x \rightarrow \infty} f(x)=0\right\} .
$$

Theorem 4.1. Let $\Phi \in \mathcal{C}(X ; \mathbb{R})$ be a strictly positive function with $\Phi^{-1} \in$ $\mathcal{C}_{0}(X ; \mathbb{R})$. Let $\mathcal{A} \subset \mathcal{C}^{\Phi}(X)$ be such that

1. $\mathcal{A}$ is a separating subalgebra of $\mathcal{C}(X ; \mathbb{R})$.

2. One has $\Phi^{-1} \in \mathcal{A}$.

Given $\varepsilon>0, \eta>0$ and $f \in \mathcal{C}(X ; \mathbb{R})$ such that $|f| \leq k \Phi^{n}$ for some $k>0$ and some $n \in \mathbb{N}$, there exists $g \in \mathcal{A} \cap \mathcal{C}^{b}(X ; \mathbb{R})$ such that

$$
|f(x)-g(x)| \leq \varepsilon \Phi^{n+\eta}(x)
$$

for all $x \in X$.

Therefore, $\mathcal{A}_{i d}$ is sequentially dense in $\mathcal{C}^{\Phi}(X)$ endowed with its $\mathcal{D F}$ topology (defined in next section). The set $\mathcal{A}_{i d}=\mathcal{A} \cap \mathcal{C}^{b}(X ; \mathbb{R})$ is a subalgebra of the Banach algebra $\mathcal{C}^{b}(X ; \mathbb{R})$. For a fixed $n \in \mathbb{N}, \Phi^{n} \mathcal{A}_{i d}$ (resp. $\left.\Phi^{n} \mathcal{C}^{b}(X ; \mathbb{R})\right)$ denotes the linear space of functions of the form $g \Phi^{n}$ where $g$ moves in $\mathcal{A}_{i d}$ (resp. in $\mathcal{C}^{b}(X ; \mathbb{R})$ ) and it is immediate that $\mathcal{A}=\bigcup_{n \geq 0} \Phi^{n} \mathcal{A}_{i d}$ (resp. $\left.\mathcal{C}^{\Phi}(X)=\bigcup_{n \geq 0} \Phi^{n} \mathcal{C}^{b}(X ; \mathbb{R})\right)$. When $\Phi$ is an unbounded function on $X$, the linear space $\Phi^{r} \mathcal{A}_{i d}$ is never an algebra, except for $r=0$. When $\Phi^{-1} \in \mathcal{A}$, the map $f \rightarrow f \Phi^{r}$ is an order-preserving linear bijection from $\mathcal{A}_{i d}$ onto $\Phi^{r} \mathcal{A}_{i d}$ for $r \in \mathbb{N}$ hence $\Phi^{r} \mathcal{A}_{i d}$ is a lattice iff $\mathcal{A}_{i d}$ has this property.

Proof. Let $\mathcal{A}_{i d}=\mathcal{A} \cap \mathcal{C}^{b}(X ; \mathbb{R})$. Using the fact that $\Phi^{-1} \in \mathcal{A} \cap \mathcal{C}_{0}(X ; \mathbb{R}) \subset$ $\mathcal{A}_{i d}$ and the fact that $\mathcal{A} \subset \mathcal{C}^{\Phi}(X)$ it is easy to see that $\mathcal{A}_{i d}$ is a separating subalgebra of $\mathcal{C}^{b}(X ; \mathbb{R})$. For each $n \in \mathbb{N}$ consider the linear space $W_{n}=$ $\Phi^{n} \mathcal{A}_{i d}$. Then $W_{n}$ is everywhere different from zero on $X$. This is clear for $n=0$, since $\Phi^{-1} \in \mathcal{A}_{i d}$ and $\Phi^{-1}(x)>0$ for all $x \in X$. Using induction on $n$, the result follows for all $n \in \mathbb{N}$. Notice that $W_{n}$ is an $\mathcal{A}_{i d}$-module, that is $\mathcal{A}_{i d} W_{n} \subset W_{n}$. By the bounded case of the weighted approximation problem (Theorem 1 of [10], p. 295) and by the remark of page 290 of [10], it follows that the $\mathcal{A}_{i d}$-module $W_{n}$ is dense in $\mathcal{C} v_{\infty}(X ; \mathbb{R})$ for any weight $v$ such that $W_{n}$ is contained in $\mathcal{C} v_{\infty}(X ; \mathbb{R})$. Now, given $f \in \mathcal{C}(X ; \mathbb{R})$ such that $|f| \leq k \Phi^{n}$ for some $k>0$ and some $n \in \mathbb{N}$, consider the weight $v=\left(\Phi^{2 n+\eta}\right)^{-1}$. Then $\Phi^{n} f$ and $W_{n}=\Phi^{n} \mathcal{A}_{i d}$ are both in $\mathcal{C} v_{\infty}(X ; \mathbb{R})$. The density of $W_{n}$ in $\mathcal{C} v_{\infty}(X ; \mathbb{R})$ implies that given $\varepsilon>0$, there is $w \in W_{n}$ such that $v(x)\left|\left(\Phi^{n} f\right)(x)-w(x)\right|<\varepsilon$ for all $x \in X$. Since $w=\Phi^{n} g$, for some $g \in \mathcal{A}_{i d}=\mathcal{A} \cap \mathcal{C}^{b}(X ; \mathbb{R})$, and $v=\left(\Phi^{2 n+\eta}\right)^{-1}$ it follows that

$$
|f(x)-g(x)| \leq \varepsilon \Phi^{n+\eta}(x)
$$

for all $x \in X$. 
Remark. In his Lemma 1 ([10], p. 294) Nachbin assumes that the algebra $\mathcal{A} \subset \mathcal{C}^{b}(X ; \mathbb{R})$ contains the constants, but this is not necessary in order to prove his Theorem 1 . Indeed, if $B$ is the subalgebra of $\mathcal{C}^{b}(X ; \mathbb{R})$ generated by $\mathcal{A}$ and $l$, then $\mathcal{A}$ and $B$ define the same equivalence classes in $X$, and any $\mathcal{A}$-module is a $B$-module, since

$$
B W \subset A W+W \subset W+W \subset W .
$$

\section{The underlying $\mathcal{D} \mathcal{F}$-topology}

Algebras of major interest are algebras of type $\mathcal{C}^{\Phi}(X)$ as studied in Theorem 4.1. This section defines a natural topology valid for many functions algebras.

Let $\left(\Phi_{i}\right), i \geq 0$ be an increasing sequence of positive continuous functions defined on the locally compact space $X$ with $\Phi_{0}=1$ the constant function. For $i \neq 0$, we drop the $\Phi_{i}$ 's belonging to $\mathcal{C}^{b}(X ; \mathbb{R})$, thus we may assume that all $\Phi_{i}$ 's are unbounded on $X$ for $i>0$ with

$$
\Phi_{0}=1 \leq \Phi_{1} \leq \Phi_{2} \leq \cdots \leq \Phi_{n} \leq \cdots .
$$

The map

$$
f \in \mathcal{C}(X ; \mathbb{R}) \rightarrow \Phi_{n} f \in \mathcal{C}(X ; \mathbb{R})
$$

restricted to $\mathcal{C}^{b}(X ; \mathbb{R})$ defines a linear bijection between $\mathcal{C}^{b}(X ; \mathbb{R})$ and its image (denoted $\Phi_{n} \mathcal{C}^{b}(X ; \mathbb{R})$ ). Since $\mathcal{C}^{b}(X ; \mathbb{R})$ is a Banach space for the supremum norm $\|\cdot\|_{\infty}$, we endow $\Phi_{n} \mathcal{C}^{b}(X ; \mathbb{R})$ with the norm

$$
\|f\|_{\Phi_{n}}=\sup \left\{\left|\frac{f(t)}{\Phi_{n}(t)}\right| ; t \in X\right\}
$$

for an element $f \in \Phi_{n} \mathcal{C}^{b}(X ; \mathbb{R})$ in order to make the map (5.1) an isometry between Banach spaces $\left(\mathcal{C}^{b}(X ; \mathbb{R}),\|\cdot\|_{\infty}\right)$ and $\left(\Phi_{n} \mathcal{C}^{b}(X ; \mathbb{R}),\|\cdot\|_{\Phi_{n}}\right)$. We put

$$
\mathcal{C}\left(X ;\left(\Phi_{j}\right)_{j \geq 0} ; \mathbb{R}\right)=\bigcup_{n \geq 0} \Phi_{n} \mathcal{C}^{b}(X ; \mathbb{R})
$$

also equal to the linear space of elements of $\mathcal{C}(X ; \mathbb{R})$ with a growth on $X$ smaller in absolute value than some homothetic of $\Phi_{i}(i$ depending on $f)$.

More generally, for a linear space $\mathcal{A} \subset \mathcal{C}\left(X ;\left(\Phi_{j}\right)_{j \geq 0} ; \mathbb{R}\right)$ such that $\Phi_{i} \in \mathcal{A}$ for all $i \geq 0$ we set

$$
\mathcal{A}_{\Phi_{n}}=\left\{f \in \mathcal{A} ; \sup _{x \in X}\left|\frac{f(x)}{\Phi_{n}(x)}\right|<+\infty\right\}
$$

therefore we get an increasing sequence of normed spaces

$$
\left(\mathcal{A}_{\Phi_{0}},\|\cdot\|_{\infty}\right) \subset\left(\mathcal{A}_{\Phi_{1}},\|\cdot\|_{\Phi_{1}}\right) \subset\left(\mathcal{A}_{\Phi_{2}},\|\cdot\|_{\Phi_{2}}\right) \cdots \subset\left(\mathcal{A}_{\Phi_{n}},\|\cdot\|_{\Phi_{n}}\right) \subset \cdots
$$


and clearly $\mathcal{A}_{\Phi_{0}}=\mathcal{A} \cap \mathcal{C}^{b}(X ; \mathbb{R}), \mathcal{A}_{\Phi_{n}}=\mathcal{A} \cap \Phi_{n} \mathcal{C}^{b}(X ; \mathbb{R})$. The norm $\|\cdot\|_{\Phi_{n}}$ choosen on $\mathcal{A}_{\Phi_{n}}$ is formula (5.2) restricted to $\mathcal{A}_{\Phi_{n}}$ therefore is the restriction to the linear space $\mathcal{A}_{\Phi_{n}}$ of the natural norm $\|\cdot\|_{\Phi_{n}}$ of $\Phi_{n} \mathcal{C}^{b}(X ; \mathbb{R})$.

Clearly, for $0 \leq i \leq j$, the identity map

$$
f \in\left(\mathcal{A}_{\Phi_{i}},\|\cdot\|_{\Phi_{i}}\right) \rightarrow f \in\left(\mathcal{A}_{\Phi_{j}},\|\cdot\|_{\Phi_{j}}\right)
$$

between normed spaces is continuous with norm $\leq 1$. We endow

$$
\mathcal{A}=\bigcup_{n \geq 0} \mathcal{A}_{\Phi_{n}}
$$

with the locally convex topology inductive limit (not strict in general) of the sequence of normed spaces $\left(\mathcal{A}_{\Phi_{j}},\|\cdot\|_{\Phi_{j}}\right)_{j \geq 0}$ : following [7], we use the notation $\varrho$ for this topology and $(\mathcal{A}, \varrho)$ for the locally convex topological vector space so defined. We note that the map (5.1) restricted to $\mathcal{A}$ is generally undefined because it sends $\mathcal{A}$ outside itself.

The unit ball of each normed space $\left(\mathcal{A}_{\Phi_{n}},\|\cdot\|_{\Phi_{n}}\right)$ is denoted $\left[-\Phi_{n}, \Phi_{n}\right]_{\mathcal{A}}$. The space $\mathcal{C}\left(X ;\left(\Phi_{j}\right)_{j \geq 0} ; \mathbb{R}\right)$ is the largest space taken into our analysis: when no ambiguity arises on the chosen sequence $\left(\Phi_{j}\right)_{j \geq 0}$, one simply write

$$
\mathcal{C}=\mathcal{C}\left(X ;\left(\Phi_{j}\right)_{j \geq 0} ; \mathbb{R}\right) .
$$

The unit ball of $\Phi_{n} \mathcal{C}^{b}(X ; \mathbb{R})=\mathcal{C}_{\Phi_{n}}$ coincides with $\left[-\Phi_{n}, \Phi_{n}\right]_{\mathcal{C}}$ and one has

$$
\left[-\Phi_{n}, \Phi_{n}\right]_{\mathcal{C}} \cap \mathcal{A}=\left[-\Phi_{n}, \Phi_{n}\right]_{\mathcal{A}}
$$

Proposition 5.1. Let $\mathcal{A}=\bigcup_{i>0} \quad \mathcal{A}_{\Phi_{i}}$ be a linear subpace of $\mathcal{C}\left(X ;\left(\Phi_{j}\right)_{j \geq 0} ; \mathbb{R}\right)$ (thus $\Phi_{i} \in \mathcal{A}$ for all $i \geq 0$ ). Under the $\varrho$-topology,

1. The locally convex topology $(\mathcal{A}, \varrho)$ is separated, bornological and quasibarrelled.

2. Any positive linear form defined on $\mathcal{A}$ is continuous for $\varrho$-topology.

3. A fundamental system of bounded subsets of $(\mathcal{A}, \varrho)$ consists of intervals $\left[-\Phi_{n}, \Phi_{n}\right]_{\mathcal{A}}$ and their homothetics.

4. The strong dual of $(\mathcal{A}, \varrho)$ is a Fréchet space.

The topology of the strong dual $\mathcal{A}^{\prime}$ of $(\mathcal{A}, \varrho)$ is the locally convex topology associated to the set of semi-norms $\|\cdot\|_{\Phi_{n}}$ defined by

$$
\|f\|_{\Phi_{n}}=\sup _{-\Phi_{n} \leq T \leq \Phi_{n}}|f(T)|
$$

for $f \in \mathcal{A}^{\prime}$. A linear map $L: \mathcal{A} \rightarrow F$ where $F$ is locally convex space is continuous if and only its restriction to each normed space $\left(\mathcal{A}_{\Phi_{n}},\|\cdot\|_{\Phi_{n}}\right)$ is continuous: this is a well-known property of inductive limits. 
Proof. Let $L$ be a positive linear form defined on $\mathcal{A}$. Each normed space $\left(\mathcal{A}_{\Phi_{n}},\|\cdot\|_{\Phi_{n}}\right)$ is an order unit space, hence the positivity of $L$ implies that $L$ restricted to $\mathcal{A}_{\Phi_{n}}$ is continuous for the norm $\|\cdot\|_{\Phi_{n}}$ implying property 2). It follows that each function $\chi_{x}$ with $x \in X$ defined by formula (5.3) is continuous. Hence, given $f \neq 0, f \in \mathcal{A}$, there exists $a \in X$ such that $f(a) \neq 0$ implying $\chi_{a}(f) \neq 0$; thus the $\varrho$-topology is separated. Assertions 1) and 4) follows from [4, Chapter 4, Part 3]. For assertion 3), due to [4, Proposition 5, p. 171], it remains to prove that each interval $\left[-\Phi_{n}, \Phi_{n}\right]_{\mathcal{A}}$ is closed. This follows from

$$
\left[-\Phi_{n}, \Phi_{n}\right]=\bigcap_{x \in X} \chi_{x}^{-1}\left(\left[-\Phi_{n}(x), \Phi_{n}(x)\right]\right) .
$$

Proposition 5.2. We assume that, for any $i \geq 0$, there exists $k \geq 0$ such that $\Phi_{i}^{2} / \Phi_{k} \in \mathcal{C}^{b}(X ; \mathbb{R})$, i.e. $\Phi_{i}^{2} \leq c_{i} \Phi_{k}$ for some $c_{i} \in \mathbb{R}$. Then:

1. $\mathcal{C} \equiv_{\text {def }} \mathcal{C}\left(X ;\left(\Phi_{j}\right)_{j \geq 0} ; \mathbb{R}\right)$ is an algebra.

2. Let $\mathcal{A}=\bigcup_{i>0} \mathcal{A}_{\Phi_{i}}$ be a subalgebra of $\mathcal{C}$ with the condition that $1 / \Phi_{i} \in$ $\mathcal{A}$ for all $i \geq 1$. Then, for $n \geq 1$, one has the equality $\mathcal{A}_{\Phi_{n}}=\Phi_{n} \mathcal{A}_{\Phi_{0}}$ and the linear normed space $\left(\mathcal{A}_{\Phi_{n}},\|\cdot\|_{\Phi_{n}}\right)$ is isometric with the normed algebra $\mathcal{A} \cap \mathcal{C}^{b}(X ; \mathbb{R})=\mathcal{A}_{\Phi_{0}}$ endowed with its supremum norm.

3. For a fixed $i$, the algebra $\mathcal{A}^{\Phi_{i}}=\bigcup_{n \geq 0} \Phi_{i}^{n} \mathcal{A}$ endowed with its $\varrho$-topology embeds continuously in $(\mathcal{A}, \varrho)$ and in $(\mathcal{C}, \varrho)$.

The importance of algebras of the form $\mathcal{A}^{\Phi}$ comes from Theorem 4.1. We always assume in the following that $\mathcal{C}$ is an algebra.

General properties of $\mathcal{C}$ can be first obtained on each $\left(\mathcal{C}^{\Phi_{i}}, \varrho\right)$ and then be gathered to $\mathcal{C}$ by property 2 ).

Proof. For 1), it suffices to prove that $f_{1} \in \mathcal{C}$ and $f_{2} \in \mathcal{C}$ imply $f_{1} f_{2} \in \mathcal{C}$. One has $f_{1} \in \mathcal{C}_{\Phi_{i_{1}}}, f_{2} \in \mathcal{C}_{\Phi_{i_{2}}}$, thus with $i=\max \left(i_{1}, i_{2}\right)$, one has $f_{1}=g_{1} \Phi_{i}$, $f_{2}=g_{2} \Phi_{i}$ with $g_{1}, g_{2} \in \mathcal{C}^{b}(X ; \mathbb{R})$. With $k$ as indicated, one has

$$
f_{1} f_{2}=g_{1} g_{2} \frac{\Phi_{i}^{2}}{\Phi_{k}} \Phi_{k}=g_{1} g_{2} g_{3} \Phi_{k} .
$$

Since $g_{3} \in \mathcal{C}^{b}(X ; \mathbb{R})$, we get $f_{1} f_{2} \in \mathcal{C}$. When $f_{1}, f_{2}$ move in $\mathcal{A}$, it follows that $g_{1}, g_{2} \in \mathcal{A}_{\Phi_{0}}$ and conversely: this shows 2$)$. Last assertion is straighforward.

For $x \in X$, let

$$
\chi_{x}: f \in \mathcal{C} \rightarrow \chi_{x}(f)=f(x) \in \mathbb{R}
$$

be the corresponding positive (continuous) linear map. 
Lemma 5.3. For $K$ compact subset of $X$, the set $\chi_{K}=\left\{\chi_{x} ; x \in K\right\}$ is an equicontinuous set of the strong dual $\mathcal{C}^{\prime}$ of $\mathcal{C}$. The linear span of the set $\bigcup_{K \in \mathbb{M}} \chi_{K}$ is dense in $\mathcal{C}^{\prime}$ for $\sigma\left(\mathcal{C}^{\prime}, \mathcal{C}\right)$ topology (here $\mathbb{M}$ stands for the family of all compact subsets of $X)$.

Proof. Since $\mathcal{C}$ is barrelled, it suffice to prove that $\chi_{K}$ is bounded on each interval $\left[-\Phi_{n}, \Phi_{n}\right]_{\mathcal{C}}$, which follows from

$$
\left|\sup _{f \in\left[-\Phi_{n}, \Phi_{n}\right]_{\mathcal{C}}} \chi_{x}(f)\right| \leq\left|\sup _{f \in\left[-\Phi_{n}, \Phi_{n}\right]_{\mathcal{C}}} f(x)\right| \leq \Phi_{n}(x) \leq \sup _{x \in K} \Phi_{n}(x)<+\infty
$$

due to continuity of $\Phi_{n}$.

The polar of $\bigcup_{K \in \mathbb{M}} \chi_{K}$ in the duality $\left\langle\mathcal{C}^{\prime}, \mathcal{C}\right\rangle$ consists of functions $f \in \mathcal{C}$ such that $\left|\left\langle\chi_{K}, f\right\rangle\right|=0$ for any compact set $K \subset X$, hence $f=0$ and our conclusion follows from the bipolar theorem.

Theorem 5.4. We assume that, for any $i \geq 0$, there exists $k \geq 0$ such that $\Phi_{i}^{2} \leq C_{i} \Phi_{k}$ for some $C_{i} \in \mathbb{R}$ and $\lim _{x \rightarrow \infty} \Phi_{i}(x)=+\infty$ for every $i \geq 1$.

Let $\mathcal{A}=\bigcup_{i \geq 0} \mathcal{A}_{\Phi_{i}}$ be a linear subspace of $\mathcal{C}$ with the condition $\Phi_{i}^{2} \in \mathcal{A}$ for every $i \geq 1$.

1. Let $\left(g_{n}\right)$ be a sequence in $\mathcal{A}$ tending to $g \in \mathcal{A}$ for $\varrho$-topology of $\mathcal{A}$. Then, $\left(g_{n}\right)$ tends to $g$ in a dominated way, i.e. there exists $k \geq 1$ and a real sequence $\varepsilon_{n} \rightarrow 0$ such that

$$
\left|g_{n}(x)-g(x)\right| \leq \varepsilon_{n} \Phi_{k}(x)
$$

for all $x \in X$ and all $n \in \mathbb{N}$.

2. Bounded subsets of $(\mathcal{A}, \varrho)$ are metrizable.

In other words, $(\mathcal{A}, \varrho)$ satisfies the strict condition of Mackey convergence [5] for sequences, due to assertion 3) of Proposition 5.1. It also follows that $\left(g_{n}\right)$ tends to $g$ in $\mathcal{C}^{\Phi_{j}}$ endowed with its $\varrho$-topology (we note that $\mathcal{C}=\bigcup_{j \geq 0} \mathcal{C}^{\Phi_{j}}$ as sets)

Proof. A convergent sequence being bounded, there exists $\lambda>0, j \in \mathbb{N}$ such that $g_{n} \in\left[-\lambda \Phi_{j}, \lambda \Phi_{j}\right]$ for all $n$. We may assume $\lambda=1$. Since $(\mathcal{A}, \varrho)$ embeds continuously in $(\mathcal{C}, \varrho)$, the sequence $\left(g_{n}\right)$ tends to $g$ for $\varrho$-topology of $\mathcal{C}$. Let $\varepsilon>0, \delta>0$ ( $\delta$ is chosen equal to 1 for any $\mathcal{A}$ and may be chosen arbitrarily small when $\Phi_{j}^{\delta} \in \mathcal{A}$ for small $\delta$, for example when $\mathcal{A}=\mathcal{C}$ ). Let $H$ be a compact set in $X$ such that $1 / \Phi_{j}^{\delta}(x) \leq \varepsilon / 2$ for $x \notin H$. One has, for $x \notin H$,

$$
\left|\frac{g_{n}(x)-g(x)}{\Phi_{j}^{1+\delta}(x)}\right| \leq \frac{\varepsilon}{2} \frac{2}{\Phi_{j}^{\delta}(x)} \leq \varepsilon .
$$


From Lemma 5.3, for every compact set $K \subset X$, one has $\left|<\chi_{K}, g_{n}-g\right\rangle$ $\mid \rightarrow 0$ as $n \rightarrow \infty$. Let $n(\varepsilon)$ such that $\left|<\chi_{H}, g_{n}-g>\right| \leq \varepsilon$ for all $n \geq n(\varepsilon)$. One has, for $x \in H$ and all $n \geq n(\varepsilon)$

$$
\left|\frac{g_{n}(x)-g(x)}{\Phi_{j}^{1+\delta}(x)}\right| \leq \varepsilon \frac{1}{\Phi_{j}^{1+\delta}(x)} \leq \varepsilon
$$

since $1 \leq \Phi_{j}$. Since there exits $k$ and $M<+\infty$ such that $\Phi_{j}^{1+\delta} \leq M \Phi_{k}$, hence combining inequalities, we get the majoration (5.4) (the majoration is precise when $\Phi_{j}^{\delta} \in \mathcal{A}$ for small $\left.\delta\right)$. The second assertion is proved in a similar way since a bounded net $\left(g_{i}\right), i \in I$ ( $I$ directed set) tending to 0 for $\varrho$-topology of $\mathcal{A}$ is contained in an homothetic of some interval $\left[-\Phi_{j}, \Phi_{j}\right]_{\mathcal{A}}$ and tends to 0 in the normed space $\left(\mathcal{A}_{\Phi_{j}^{2}},\|\cdot\|_{\Phi_{j}^{2}}\right)$; therefore, on $\left[-\Phi_{j}, \Phi_{j}\right]_{\mathcal{A}}$, topologies induced by $(\mathcal{A}, \varrho)$ and $\left(\mathcal{A}_{\Phi_{j}^{2}},\|\cdot\|_{\Phi_{j}^{2}}\right)$ are the same.

Corollary 5.5. Let $\mathcal{A}=\bigcup_{i \geq 0} \mathcal{A}_{\Phi_{i}}$ be a subalgebra of $\mathcal{C}$. Convergent sequences in $(\mathcal{A}, \varrho)$ are dominated convergent sequences.

\section{References}

[1] Bidegain, F., Pinczon, G., Quantization of Poisson-Lie Groups and Applications, Comm. Math. Phys. 179 (1996), 295-332.

[2] Brosowski, B., Deutsch, F., An elementary proof of the Stone-Weierstrass theorem, Proc. Amer. Math. Soc. 81 (1981), 89-92.

[3] Fan, K., Minimax theorems, Proc. Nat. Acad. Sci. U.S.A. 39 (1953), 42-47.

[4] Grothendieck, A., Topological Vector Spaces, Gordon and Breach, New York, 1973.

[5] Grothendieck, A., Sur les espaces $(\mathcal{F})$ et $(\mathcal{D} \mathcal{F})$, Summa Brasil. Math. 3(6) (1954), $57-123$.

[6] Jurzak, J.-P., Un aspect fonctionnel de l'intégration, C. R. Acad. Sci. Paris Ser. I Math. 296 (1983), 589-591.

[7] Jurzak, J.-P., Unbounded Non-Commutative Integration, Math. Phys. Stud. 7, Kluwer Acad. Publ., Dordrecht, 1985.

[8] Kindler, J., Topological minimax theorems and approximation, J. Math. Anal. Appl. 210 (1997), 8-21.

[9] Machado, S., On Bishop's generalisation of the Weierstrass-Stone theorem, Indag. Math. 39 (1977), 218-224.

[10] Nachbin, L., Weighted approximation for algebras and aodules of continuous functions: real and self-adjoint complex cases, Ann. of Math. 81 (1965), 289-302.

[11] Prolla, J. B., On the Weierstrass-Stone theorem, J. Approx. Theory 78 (1994), 299313.

[12] Ransford, T. J., A short elementary proof of the Bishop-Stone-Weierstrass theorem, Math. Proc. Cambridge Philos. Soc. 96 (1984), 309-311.

[13] Sion, M., On general minimax theorems, Pacific J. Math. 8 (1958), 171-176. 
JEAN-PAUL JURZAK

Laboratoire Gevrey de Mathématique Physique

Université DE Bourgogne

FaCulté des Sciences et TeChniques

BP 47870, 21078 DiJOn CEDEX

FRANCE

E-MAIL: JEAN-PAUL.JURZAK@U-BOURGOGNE.FR 